\title{
Modelado del comportamiento mecánico de unión socket - muñón, en amputados de miembro inferior
}

\author{
José Alejandro Guerrero Vargas`, Diego Pataquiva Wilches \\ Grupo de Investigación de Diseño Avanzado, Fundación Universidad de América. \\ Bogotá, Colombia
}

FECha de ENTREga: 30 DE MAYo De 2014

FECHA DE EVAluACión: 26 DE AGOSTO DE 2014

FECHA DE APROBACIÓN: 4 DE SEPTIEMBRE

\begin{abstract}
Resumen Se determinó, por medio del método de elementos finitos, el comportamiento de la unión muñón - socket, en una amputación trans - tibial; el dominio del problema contempla la piel, el socket y el hueso como elementos en contacto. Para ello, se generaron los moldes físicos en un caso específico (paciente), se obtuvieron los modelos geométricos CAD, se realizó el análisis biomecánico en la interfaz, tanto en bipedestación como en marcha, y se llevó a cabo la simulación en ANSYS Workbench. Con base en los resultados obtenidos se establecieron los puntos críticos de presión en comparación con el umbral de dolor. De esta forma, se brinda información para que, al diseñar el socket, se busque una mejor distribución de esfuerzos en la interfaz y, con ello, una mejor calidad de vida del paciente.
\end{abstract}

Abstract It was possible to determine the behavior of the stump/socket junction in a transtibial amputation by using the finite element method. The area of interest includes the skin, the socket, and the bone as elements in contact. Therefore, we generated physical molds for a specific case (patient), resulting in CAD geometric modeling. Also, we performed the biomechanical analysis on the interface - both standing and walkingand carried out the ANSYS Workbench simulation. Based on the results, we were able to establish the critical pressure points compared to the pain threshold. This way, we were able to provide information in order to better distribute the interface efforts and at the same time, generate an improved quality of life to the patient when designing the socket.

Palabras Clave: análisis de elementos finitos, interfaz socket - muñón, calidad de vida, umbral de dolor.

Keywords: finite element analysis, stump/socket interface, quality of life, pain threshold.

\footnotetext{
* Docente investigador, Fundación Universidad de América, Colombia.
} jose.guerrero@investigadores .uamerica.edu.co 


\section{Introducción}

De una manera general la biomecánica puede definirse como la rama de la ciencia que se encarga de evaluar las fuerzas, y sus efectos, en sistemas que tienen vida [1]; Sin embargo, cuando recurrimos a un término enfocado en el ser humano, se hace referencia a una ciencia interdisciplinar que estudia el movimiento del cuerpo [2]. Parte de la labor en la que se ocupa la biomecánica está encaminada a tratamientos de rehabilitación sobre pacientes que, por situaciones de violencia o a causa de enfermedad natural, pierden alguna parte de su cuerpo. Como resultado, existen diversos análisis sobre dispositivos protésicos, mecanismos artificiales que reemplazan miembros faltantes y que se espera suplan las funciones que se vieron afectadas por la pérdida [3]; sobre el muñón, o parte del miembro cortado que permanece adherida al cuerpo [3]; y sobre el encaje o socket, encargado de realizar la conexión entre el dispositivo protésico y el muñón.

Las amputaciones datan desde la prehistoria y, sobre el 484 A.C., se reporta la primera prótesis que fue empleada por una persona, un dispositivo de madera que suplía parte de la extremidad inferior; luego de esto, diversos aportes históricos han contribuido al desarrollo y evolución de las prótesis a los dispositivos que hoy en día se conocen [4]. En la actualidad existen múltiples estudios en prótesis de miembro inferior, análisis del uso de sensores empleados en estos dispositivos [5], análisis de elementos finitos [6], análisis de umbral de dolor [7], entre otros.

En Colombia, la demanda de prótesis es considerable debido a los factores de violencia, enfermedades naturales, accidentes de tránsito o laborales, entre otros. De acuerdo con el Programa Presidencial para la Acción Integral contra Minas Antipersonal, durante el período de 1990 a Mayo de 2014, se presentaron 10751 víctimas a causa de minas antipersonal (MAP) y municiones sin explotar (MUSE) de los cuales el $80 \%$ resultaron heridos y el $20 \%$ falleció; es decir que, de esta población, existen 8572 personas que potencialmente pueden sufrir amputación [8]. Adicional a esto, según el Ministerio de Protección Social, entre un 7.4 y $9.4 \%$ de la población adulta es diabética, lo que equivale, en cifras del 2011, alrededor de 1.7 millones de colombianos y, sobre este número de individuos, se estima que el $12 \%$ requiere de una amputación; un aproximado de 204000 personas [9]. En conclusión, se puede observar la importancia de este tipo de estudios en Colombia, siendo el mayor causal de esta situación la diabetes.

\section{Materiales y métodos}

El estudio presentado en este documento se basó en la morfología y características de un paciente masculino de $1.69 \mathrm{~m}$ y $66 \mathrm{~kg}$ cuya patología corresponde a diabetes. Los modelos geométricos del muñón se generaron a partir de una tomografía computarizada del paciente que después fue reconstruida como se observa en la figura 1.

En el caso del socket, el desarrollo del modelo geométrico partió de un molde, o negativo del muñón, el cual es recubierto con una capa de yeso para generar la forma del socket. Luego de esto, se realizó un escaneo láser y, de esta forma, se 


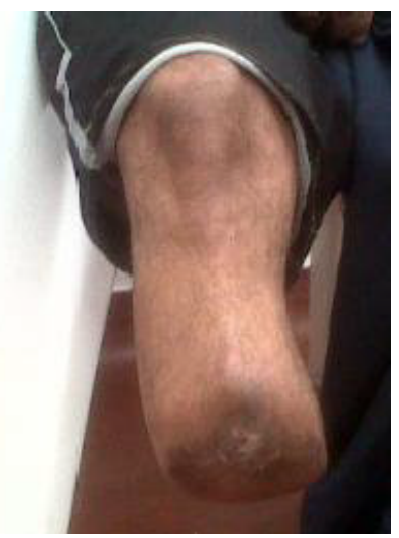

(a) Muñón paciente caso de estudio.

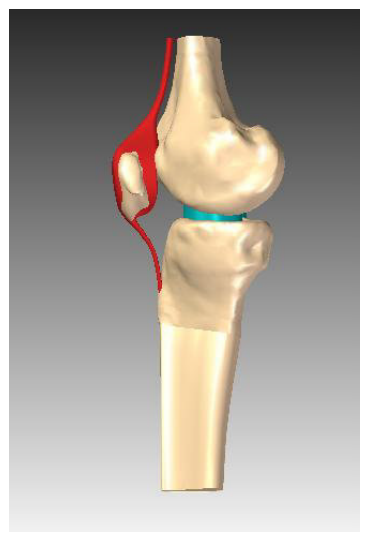

(b) Estructura ósea reconstruida a partir de la tomografía computarizada del paciente.

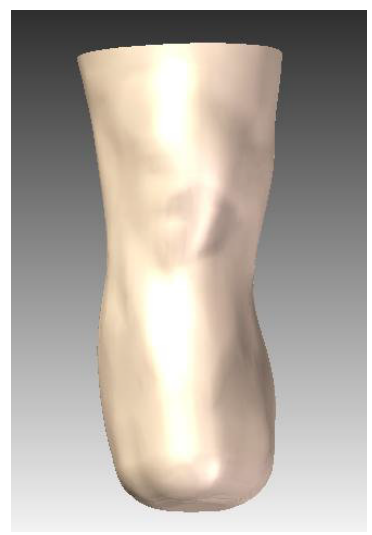

(c) Tejido blando reconstruido a partir de la tomografía computarizada del paciente.

\section{Figura 1.}

obtuvo la nube de puntos que permitió la implementación del modelo CAD 3D (Figura 2).

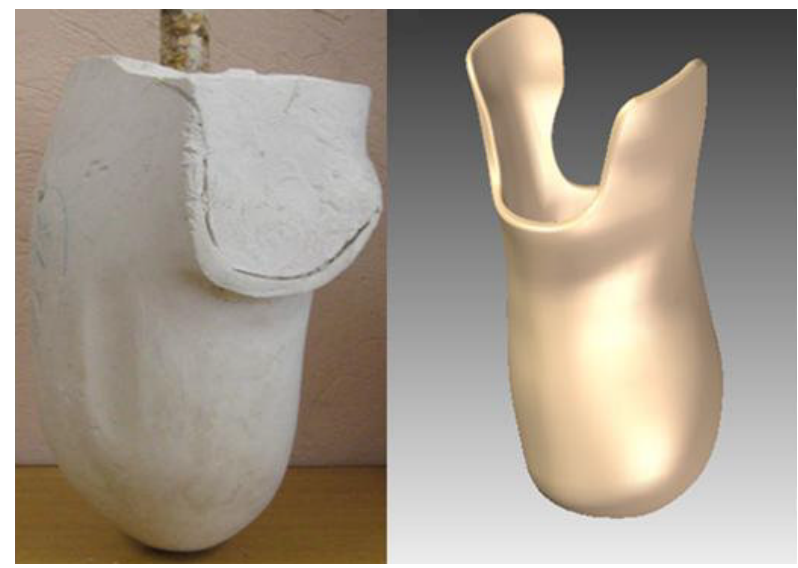

Figura 2. Molde de yeso para el escaneo 3D y modelo CAD 3D del socket.

Una vez obtenido el modelo geométrico se procedió al análisis biomecánico. El ser humano, durante su actividad diaria, puede realizar labores como caminar, trotar, subir escalera, marchar, entre otras; sin embargo, cuando una persona ha sufrido algún tipo de amputación, y el paciente es una persona que no desarrolla 
actividades como competencia de alto nivel, son de interés en particular dos: la bipedestación y la marcha. La primera corresponde a la capacidad de mantenerse, en los dos pies, erguido [6]. La segunda hace alusión al movimiento de las extremidades inferiores para realizar el desplazamiento. Dicha actividad se realiza de un modo semiautomático fruto de un aprendizaje bien definido [10,11]. La marcha humana se puede subdividir en ciclos que se componen de dos pasos, comienza con el apoyo del talón de un pie en el suelo y termina cuando se vuelve a presentar dicho apoyo con el mismo pie (Figura 3). Cada ciclo se compone de una fase de apoyo y una fase de balanceo $[6,10]$.

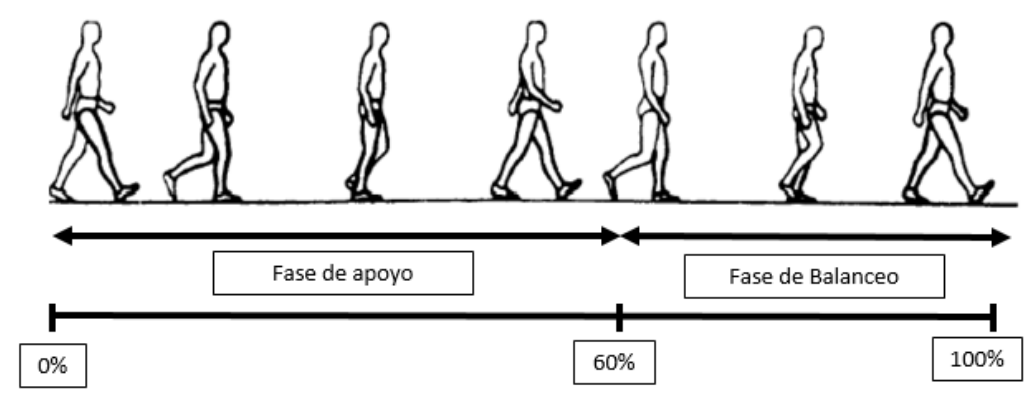

Figura 3. Ciclo de marcha humana. Adaptado de [10].

Para la etapa de bipedestación, el análisis se llevó a cabo con el peso total del paciente dividido en dos. Por su parte, en marcha, el análisis se desarrolló en la fase de apoyo y en la primera etapa de la fase de balanceo; esto debido a que es en estos períodos donde el pie se encuentra en contacto con el suelo y, por ende, es el momento en que mayores esfuerzos percibe la extremidad. La fase de apoyo comprende aproximadamente el $60 \%$ del ciclo total y está compuesta de cuatro etapas: contacto inicial $(0-2 \%)$, respuesta a la carga $(0-10 \%)$ y apoyo medio $(10-30 \%)$, contacto final $(30-50 \%)$ [12].

Después de haber definido las etapas dónde se enfocara el estudio, es necesario determinar cuáles son los músculos que interfieren en el análisis. De acuerdo con Javier Daza [13], y adecuando su concepto a la condición de amputación del caso de estudio, los músculos que tienen una influencia significativa en la marcha, ya que son los que generan el avance, corresponden al recto femoral, vasto medial, vasto lateral, vasto intermedio, bíceps femoral (cabeza larga y cabeza corta), semimembranoso y semitendinoso. Con esto, y con la ayuda del software OpenSim [14] se procede al cálculo de cargas y momentos totales presentes en cada una de las etapas en la que se genera apoyo durante la marcha.

Por último, se procede a ingresar los modelos geométricos, las cargas, los momentos y las restricciones en el software Ansys Workbench donde se llevó a cabo el análisis por elementos finitos (Figura 4). 


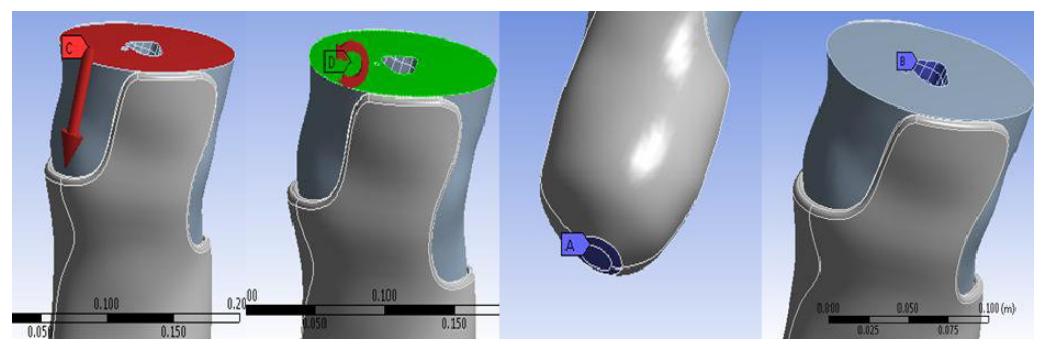

Figura 4. Condiciones de frontera para los análisis estructurales implementados. En rojo fuerzas y momentos aplicados; en azul las restricciones.

En la simulación, los materiales se definieron como lineales, homogéneos e isotrópicos y el análisis correspondió a un análisis estructural estático para las cinco etapas. Las propiedades de material empleadas se obtuvieron de la literatura y corresponden a las establecidas en el cuadro 1.

\begin{tabular}{ccc}
\hline Elemento & Módulo de Young (MPa) Coeficiente de Poisson \\
\hline Conjunto óseo [15] & 100000 & 0.3 \\
Menisco [16] & 250 & 0.45 \\
Tendón [17] & 15 & 0.47 \\
Tejido Blando [15] & 0.2 & 0.49 \\
socket [15] & 0.38 & 0.39 \\
\hline
\end{tabular}

Cuadro 1. Propiedades mecánicas de los materiales empleados.

\section{Análisis de los resultados}

De acuerdo con Lee et. al. [15], quien por medio de un indentador, aplicado a pacientes, determinó los esfuerzos máximos que soporta un ser humano en las diferentes regiones del muñón (Figura 5), dichos valores corresponden al umbral del dolor. En este trabajo, fueron empleados como valores límites de esfuerzos en la relación existente entre el muñón y el socket para establecer su buen funcionamiento; sin embargo, es inapropiado considerar que superar el umbral de dolor reportado por [15] corresponde a un mal funcionamiento del socket ya que el estudio se realizó con cargas puntuales y sin tener en cuenta que las personas difieren en su tolerancia al dolor.

En la figura 6 se observan los esfuerzos resultantes presentes en bipedestación. Se obtuvo un esfuerzo mínimo de $194.53 \mathrm{~Pa}$ y un esfuerzo máximo de $113.3 \mathrm{KPa}$ 

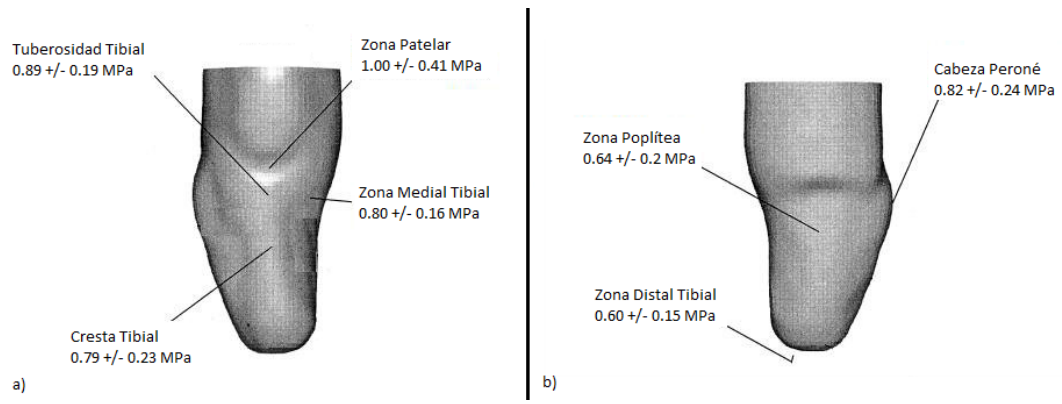

Figura 5. Regiones del muñón. a) Vista anterior. b) Vista posterior. Adaptado de [15].

en la zona distal tibial. En general, se puede apreciar que, durante esta etapa, el comportamiento de la unión muñón - socket es adecuado ya que el umbral de dolor no es superado en ninguna de las regiones preestablecidas.

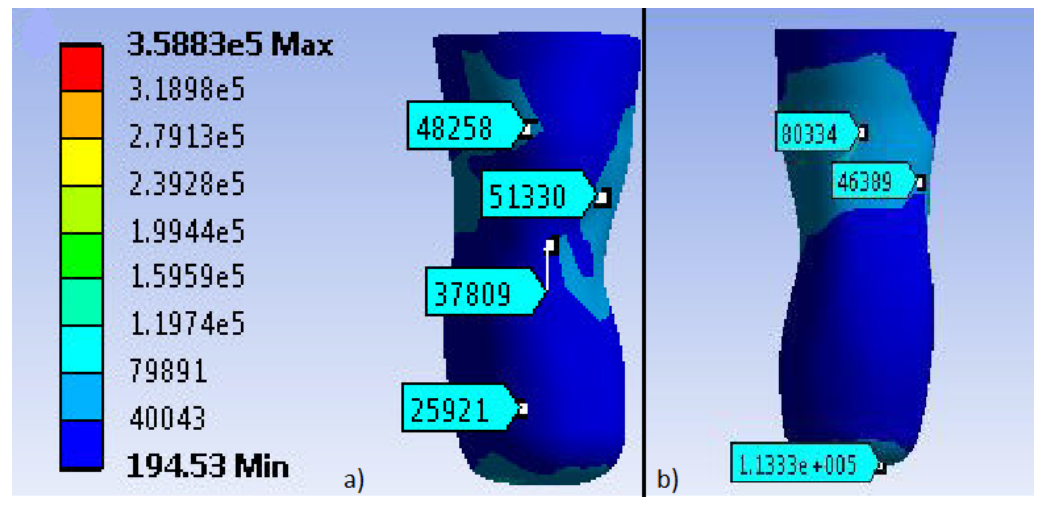

Figura 6. Esfuerzos mecánicos sobre el muñón en bipedestación (MPa). a) Vista anterior. b) Vista posterior.

Por su parte, las figuras 7 a 11 se observan los esfuerzos obtenidos para las diferentes etapas de la fase de apoyo de la marcha humana. Los rangos de esfuerzos sobre el muñón fueron de 495.94 Pa a 1.41 Mpa, de 498.09 Pa a 1.25 $\mathrm{Mpa}$, de $817.1 \mathrm{~Pa}$ a $1.29 \mathrm{Mpa}$, de $797.49 \mathrm{~Pa}$ a $1.56 \mathrm{Mpa}$ y de 285.03 a $1.97 \mathrm{Mpa}$ para contacto inicial, respuesta a la carga, apoyo medio, apoyo final y prebalanceo, respectivamente. En todos los casos, el esfuerzo máximo se presentó en el extremo de la zona distal tibial; considerando esta región como un punto crítico para el futuro diseño de sockets trans - tibiales. Es de resaltar que los esfuerzos máximos que se visualizan en la regleta de colores corresponden a valores obtenidos en 
la región de contacto con el componente óseo pero, bajo los parámetros de este trabajo, no son considerados criterios de buen o mal comportamiento de la unión socket - muñón.

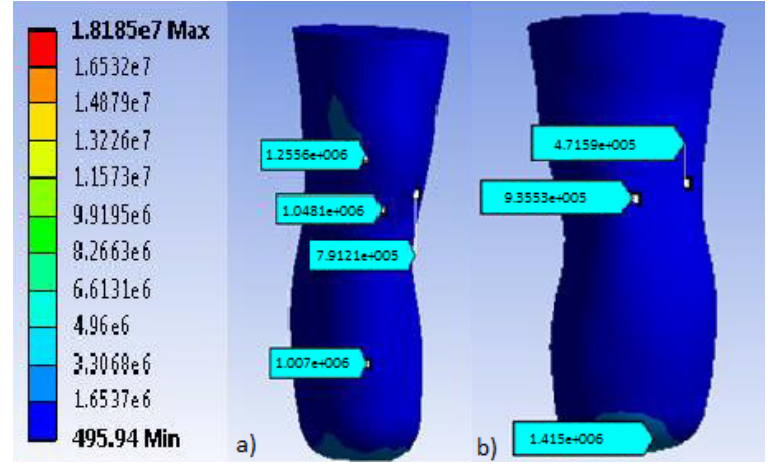

Figura 7. Contacto inicial

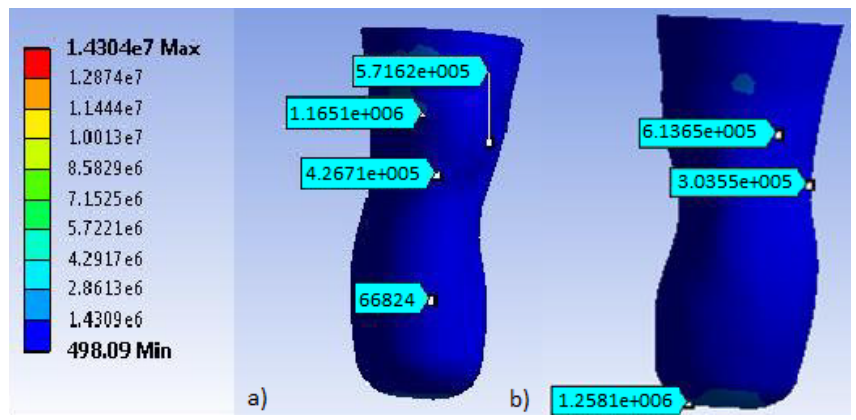

Figura 8. Respuesta a la carga

\section{Conclusiones}

Como se aprecia en los resultados, existen zonas críticas donde se presentan concentraciones considerables de esfuerzos como lo son la zona patelar y la zona distal tibial; por otra parte, también se observan regiones en las que los esfuerzos no son considerables como las zonas medial, la cabeza del peroné y la cresta tibial. Se puede pensar en dispositivos de encaje protésico que permitan una mejor 


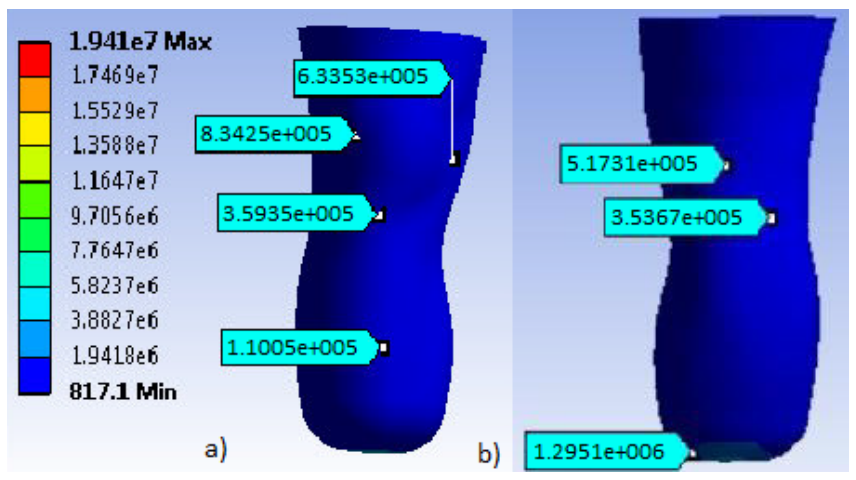

Figura 9. Apoyo medio

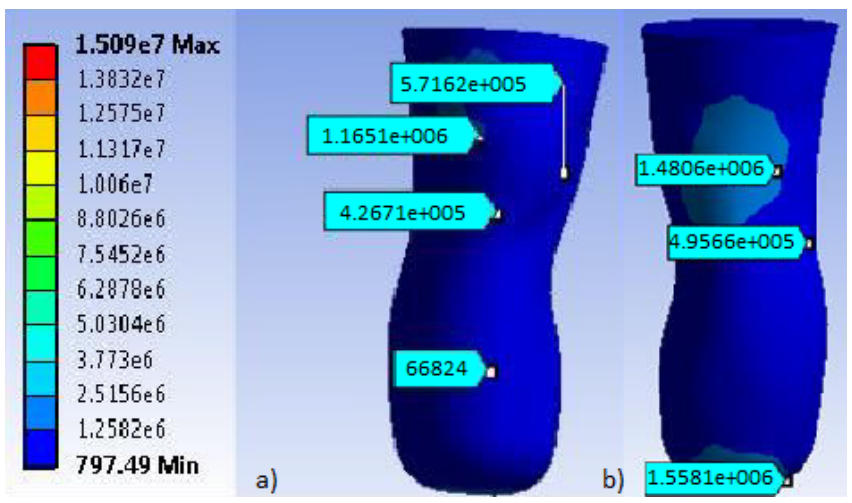

Figura 10. Apoyo final

distribución de esfuerzos y, de esta forma, disminuyan los puntos de concentración, garantizando que en las zonas del muñón los esfuerzos no superen umbrales de dolor.

\section{Referencias}

1. McGinnis, P.: Biomechanic of sport and exercise, Champaign, Illinois: Human Kinetics, (2013).

2. Winter, D.: Biomechanics and motor control of human movement, Hoboken, Nueva Jersey: John Wiley \& Sons, Inc, (2009).

3. Real Academia Española: Diccionario de la lengua española, (2001).

4. Sellegren, K.: An early history of lower limb amputations and prostheses, The Iowa Orthopaedic Journal, vol. 2, pp. 13-27, (1982). 


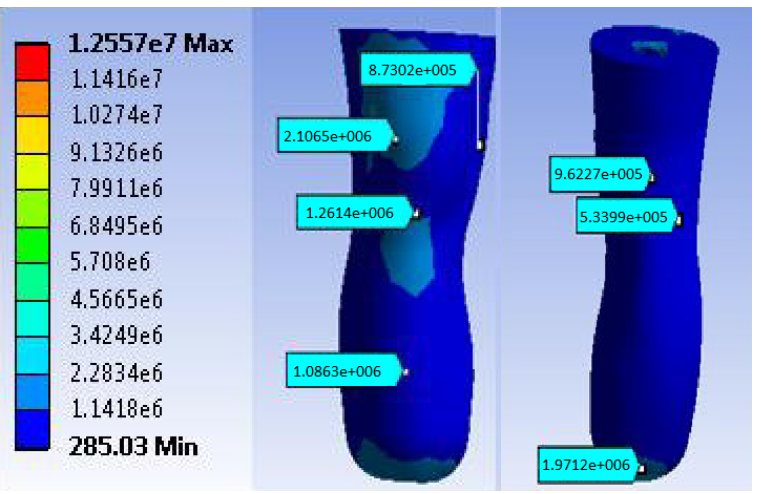

Figura 11. Prebalanceo

5. Oguz Kapti, A., Muhurcu G.: Wearable acceleration sensor application in unilateral trans-tibial amputation prostheses, Biocybernetics and Biomedical Engineering, vol. 34, pp. 53-62, (2014).

6. Pataquiva, D.: Modelado del comportamiento mecánico de unión socket - muñón, en amputados de miembro inferior, Bogotá, D.C.: Fundación Universidad de América, (2013).

7. Ramírez Patiño, J. F.: Nivel de confort y distribución de esfuerzos en la interfaz socket - muñón en amputados transfemorales, Medellín: Universidad Nacional de Colombia, (2011).

8. Departamento Administrativo de la Presidencia de la República, Programa Presidencial para la Acción Integral contra Minas Antipersonal, 16 Junio 2014. [En línea]. disponible: http://www.accioncontraminas.gov.co/.

9. Ministerio de Salud la Protección Social, Presentación 1319 Direcciones [diapositivas de PowerPoint], 2014 Junio 16. [En línea]. disponible: https://docs.google.com/ presentation/d/1ivdT8FnkB70qfqNU1KDolCdQgsywZr5Hm2q89DUqUA0/embed?hl= es\&size $=$ m\#slide=id.p34.

10. Wiereszen, N.: Análisis de la actividad muscular en posición bípeda y durante la marcha, Bilbao: Universidad del País Vasco Euskal Herriko Unibertsitatea, (2005).

11. Lara M., Angulo M., Llanos M.: Actividad electromiográfica normal en la marcha humana, Biomecánica, vol. 7, pp. 110-116, (1996).

12. Perry, J.: Gait analysis: normal and pathological function, Journal of Pediatric Orthopaedics, vol. 12, no 6, p. 815, (1992).

13. Daza Lesmes, J.: Evaluación clínico - funcional del movimiento corporal humano, Bogotá: Editorial Médica Internacional, (2007).

14. Standford University: OpenSim, Standford University, Palo Alto, (2007).

15. Jia, X., Zhang M., Lee, W.C.C.: Load transfer mechanics between trans-tibial prosthetic socket and residual limb-dynamic effects, Journal of Biomechanics, vol. 37, no 9, pp. 1371-1377, (2004).

16. Papaioannou, G., Beillas, P., Anderst, W., Yang K., Tashman, S.: A new method to investigate in vivo knee behavior using a finite element, Journal of biomechanics., vol. 37, no 7, pp. 1019 - 1030, (2004).

17. Wang, J.: Mechanobiology of tendon, Journal of Biomechanics, vol. 39, no 9, pp. 1563 - 1582, (2006). 